\title{
MicroRNA-146a and -21 cooperate to regulate vascular smooth muscle cell proliferation via modulation of the Notch signaling pathway
}

\author{
JIAN CAO, KUI ZHANG, JUBING ZHENG and RAN DONG
}

Department of Cardiac Surgery, Beijing Anzhen Hospital Affiliated to Capital Medical University, Bejing 100029, P.R. China

Received January 16, 2014; Accepted November 3, 2014

DOI: $10.3892 / \mathrm{mmr} .2014 .3107$

\begin{abstract}
A number of microRNAs (miRs) have been shown to participate in the regulation of vascular smooth muscle cell (VSMC) proliferation, a key step in the formation of atherosclerotic plaque, by targeting certain genes. The aim of the present study was to investigate the roles of miR-146a and miR-21 in VSMC growth and to study the underlying mechanisms. The expression levels of four previously reported, differentially expressed microRNAs in atherosclerotic plaque (miR-146a/b, miR-21, miR-34a and miR-210) were measured in two groups: An atherosclerotic plaque group $(n=10)$ and a normal control group $(n=10)$. Polymerase chain reaction $(P C R)$ analysis revealed that the relative expression levels of miR-146a and miR-21 in atherosclerotic plaque samples were significantly upregulated to $\sim 260$ and $250 \%$, respectively, compared with those in normal controls. Notch2 and Jag1 were confirmed to be target genes of miR-146a and miR-21 through the use of a luciferase assay, PCR and western blot analysis. Additionally, VSMCs transfected with miR-146a expressed significantly lower levels of Notch2 protein and presented an accelerated cell proliferation, which could be attributed to a reduction in the levels of cell cycle arrest. Cotransfection of miR-146a and miR-21 further promoted cell cycle progression in addition to VSMC proliferation. In conclusion, the present study revealed that miR-146a and miR-21 were significantly upregulated in atherosclerotic plaque, and cooperated to accelerate VSMC growth and cell cycle progression by targeting Notch 2 and Jag1.
\end{abstract}

\section{Introduction}

The Notch signaling pathway is highly conserved throughout evolution, and it has a critical role in intercellular signaling communication. The Notch signaling pathway was initially identified in Drosophila, where a mutant allele caused a notched

Correspondence to: Dr Ran Dong, Department of Cardiac Surgery, Beijing Anzhen Hospital Affiliated to Capital Medical University, 2 Anzhen Rd, Beijing 100029, P.R. China

E-mail: DongR_22@163.com

Key words: microRNA-146a, microRNA-21, vascular smooth muscle cell, Notch2, Jag1 wing, and is considered to be a crucial regulator during the embryonic development of vertebrate and in invertebrate organisms (1). Thus far, four Notch receptors (Notch1-4) and five Notch ligands (Jag1 and Jag2 from the Jagged/serrate family and Delta-like ligands (DLL1, DLL3 and DLL4) have been identified in mammals, and different receptor-ligand combinations may generate distinct responses (2). In addition, Notch is functionally involved in regulating a number of cellular activities, including differentiation, apoptosis and proliferation in a wide range of cell types during adult life (3). Vascular smooth muscle cells (VSMCs) have an important role in the formation of the fibrous cap in advanced atherosclerosis (4), and increased proliferation of VSMCs in the media and intima of vessels has been shown to lead to VSMC accumulation and vascular remodeling (5). While the Notch signaling pathway is clearly involved in regulating the proliferation of VSMCs, the specific role of each family member remains controversial. It has been reported that Notch1 is a positive regulator of the growth of VSMCs in response to vascular injury (6). However, another study indicated that Notch2 caused cell cycle arrest in VSMCs following carotid ligation (7).

MicroRNAs (miRs) are a class of non-protein-coding RNAs that are 22 nucleotides long, which are able to regulate gene expression by binding the 3'-untranslated region (3'-UTR) of the messenger RNA (mRNA) of the target gene, resulting in translational repression and/or mRNA degradation (8). It is generally accepted that $\sim 1 / 3$ human genes are regulated by $\mathrm{miR}(9)$, and that miRs have important roles in cellular activities, including cell proliferation, apoptosis and immune response (10-12). Thus, the regulatory network of miRs has attracted the interest of researchers. It has previously been reported that miRs participate in the phenotypic control of VSMCs $(13,14)$. Raitoharju et al $(15)$ have created a thorough comparative screen by using a microarray assay to identify the differentially expressed miRs in samples from atherosclerotic plaque and controls, identifying miR-146a/b, miR-21, miR-34a and miR-210 as potential candidate miRs responsible for the differing vascular phenotype. In the present study, the upregulation of miR-146a and miR-21 was confirmed in atherosclerotic plaque, while the expression levels of miR-34a and miR-128 were found to be similar. Subsequently, by searching miR databases and using in silico pathway/function analysis, Notch1 and Notch2 were identified as potential target genes of miR-146a, which concurs with a previous study that determined Notch1 to be a target of miR-146a in glioma cells (16). Of note, Notch2 has 
been confirmed to be a negative regulator of VSMC proliferation (6), while Notch1 is a positive regulator (7). The aim of the present study was to investigate the conflicting roles of Notch1 and Notch2 in the regulation of VSMC behavior and confirm their function and their net effect within cells.

The present study aimed to confirm Notch1/Notch2 and Jag1 as direct target genes of miR-146a and miR-21 respectively in VSMCs, and examine the effect of miR-146 and miR-21 on the proliferation of VSMCs and cell cycle control.

\section{Materials and methods}

Study sample. The atherosclerotic vascular samples were collected from patients that underwent a carotid endarterectomy (defined as "case", $\mathrm{n}=10$ ) and the reference left internal mammary arteries were obtained during coronary artery bypass surgery (defined as "control", $\mathrm{n}=10$ ). All surgical procedures were performed at the Department of Cardiac Surgery, Beijing Anzhen Hospital Affiliated to Capital Medical University (Bejing, China), and written informed consent was obtained from each participant. The study was approved by the Ethics Committee of Capital Medical University and the clinical investigation followed the principles of the Helsinki declaration. Demographics and clinicopathological characteristics are presented in Table I.

$R N A$ preparation and quantitative reverse transcription polymerase chain reaction ( $q R T-P C R)$. Total RNA, including miR, was extracted using TRIzol (Invitrogen Life Technologies, Carlsbad, CA, USA), and miR expression was determined by qRT-PCR through the use of the Universal cDNA Synthesis and SYBR Green Master Mix kits (Applied Biosystems, Foster City, CA, USA). Quantitative PCR of miR-146a/b, miR-21, miR-34a and miR-210 was performed in an ABI PRISM 7700 Sequence Detection System (Applied Biosystems). As an internal control, U6 primers were used for RNA template normalization. The primer sequences used for the quantitative PCR are listed in Table II. cDNA fragments were denatured at $95^{\circ} \mathrm{C}$ for $15 \mathrm{sec}$, annealed at $58^{\circ} \mathrm{C}$ for $15 \mathrm{sec}$ and extended at $72^{\circ} \mathrm{C}$ for $45 \mathrm{sec}$, for 40 cycles. Primers were synthesized in Shanghai Gemma, Co., Ltd. (Shanghai, China). All PCR experiments were performed in triplicate, and the differences were calculated using the $\Delta \Delta \mathrm{Ct}$ method.

Cell culture. All experiments were conducted using human coronary artery smooth muscle cells (HCASMCs), which were purchased from the Shanghai Cell Bank of the Chinese Academy of Sciences (Shanghai, China) and were cultured in HCASMC PROLIF (M231/SMGS; Invitrogen Life Technologies) supplemented with $10 \%$ fetal bovine serum (Invitrogen Life Technologies), and 1\% streptomycin/penicillin at $37^{\circ} \mathrm{C}$ in a humidified $5 \% \mathrm{CO}_{2}$-containing atmosphere.

Luciferase assay. HCASMCs were grown to $70 \%$ confluence and transfected in triplicate with either a firefly luciferase reporter plasmid containing the 3'-UTR of Notch1 or Notch2 or a Renilla luciferase reporter control vector (pRL-TK). The cells were harvested after $24 \mathrm{~h}$ and the activities of both firefly and Renilla luciferases were measured with the LB 955 Luminometer system using the Dual Luciferase Reporter system (Promega, Madison, WI, USA), according to the manufacturer's instructions. The activity of firefly luciferase was normalized to that of Renilla luciferase.

Transfection with miR-146a, miR-21 mimics and inhibitors. The miR-146a, miR-21, miR-128 and miR-34a mimics and the negative control (NC, a scramble non-specific sequence without a known human target) (Genepharma, Shanghai China) were diluted in the medium to a final concentration of $100 \mathrm{nmol} / \mathrm{l}$. Cells were incubated with the mimics and NC for $48 \mathrm{~h}$ using Transfectamin 2000, according to the manufacture's instructions (Invitrogen Life Technologies). Efficiency of transfection was determined by comparison against the NC provided by Genepharma.

MTT assay. HCASMCs were transfected with miR-146a and/or miR-21 mimics or NC and incubated for $48 \mathrm{~h}$. A $0.5 \%$ solution of MTT $(20 \mu \mathrm{l})$ was added to each well and the cells incubated for a further $4 \mathrm{~h}$. The medium was subsequently replaced with $150 \mu \mathrm{l}$ dimethyl sulfoxide (DMSO; Sigma-Aldrich, St. Louis, MO, USA) prior to being added to the microplate. The survival rate was determined by measuring the optical density (OD) values at a wavelength of $492 \mathrm{~nm}$ wavelength and comparing with the OD of the control (Spectrophotometer, Jenway Ltd., Essex, UK).

Flow cytometric analysis. Transfected cells were typsinized, washed twice with phosphate-buffered saline (PBS) and fixed with $70 \%$ ethanol on ice for $1 \mathrm{~h}$. The fixed cells were centrifuged and resuspended in PBS at a density of $1 \times 10^{6}$ cells $/ \mathrm{ml}$. Following a 30-min incubation with ribonuclease (RNase A) at a final concentration of $3,000 \mathrm{U} / \mathrm{ml}$ at $37^{\circ} \mathrm{C}$, trypsinized cells were filtered through a nylon mesh (BD Biosciences, USA). The cell suspension was stained with propidium iodide prior to analysis on a flow cytometer (FACSCanto II, BD Biosciences).

Western blot analysis. HCASMCs were transfected with the mimics and inhibitors of miR-146a, miR-21 and the controls. Following transfection for 72-h, cells were lysed on ice in lysis buffer (Beyotime, China). Cell lysates were loaded onto $10 \%$ SDS-polyacrylamide gels for electrophoresis, and the separated proteins were transferred onto a polyvinylidene fluoride membrane (EMD Millipore, Billerica, MA, USA), which was subsequently blocked with tris buffered saline with Tween 20, containing 5\% non-fat dry milk for $1 \mathrm{~h}$ at room temperature. The membrane was subsequently incubated with the anti-Notch1 antibody, anti-Notch2 antibody and anti-Jag1 antibody $(1: 1,500)$. All the antibodies were polyclonal, raised in rabbit and obtained from Santa Cruz Biotechnology, Inc. (Dallas, TX, USA) at $4^{\circ} \mathrm{C}$ overnight, followed by incubation with horseradish peroxidase-conjugated anti-rabbit secondary antibody (Santa Cruz Biotechnology, Inc.) at room temperature for $1 \mathrm{~h}$. Chemical fluorescence was detected using an enhanced chemoluminescence kit (GE Healthcare, Little Chalfont, UK) according to the manufacturer's instructions. The target bands were densitometrically analyzed and normalized to GAPDH. The ratio of target protein/GAPDH was used for semi-quantification and comparison between the different groups.

Transwell invasion and migration assays. A Transwell insert invasion assay was performed in a 24-well plate fitted with 
Table I. Clinical characteristics of the controls and cases recruited in this study.

\begin{tabular}{lccr}
\hline Variable & Controls $(\mathrm{n}=10)$ & Cases $(\mathrm{n}=10)$ & P-value \\
\hline Age (years) & $56.6 \pm 5.5$ & $58.1 \pm 4.9$ & 0.527 \\
Gender (M/F) & $7 / 3$ & $6 / 4$ & 0.640 \\
Blood pressure (mmHg) & & & \\
Systolic & $146 \pm 11.2$ & $149 \pm 12.1$ & 0.572 \\
Diastolic & $93 \pm 10.4$ & $95 \pm 10.1$ & 0.668 \\
Glucose (mg/dl) & $138 \pm 15.2$ & $144 \pm 12.3$ & 0.345 \\
Body mass index $\left(\mathrm{kg} / \mathrm{m}^{2}\right)$ & $27.8 \pm 4.1$ & $28.9 \pm 4.5$ & 0.419
\end{tabular}

Values are expressed as the mean \pm standard deviation. M, male; F, female.

insert membranes (8-mm pore size; Costar, Corning, NY, USA). As described previously (17), cell invasion was examined using polycarbonate membrane cell culture inserts (Costar) coated with growth factor reduced Matrigel (BD Biosciences, Bedford, MA, USA). The cells transfected with negative control, or miR-146a or miR-21 mimics or inhibitors, were treated with $10 \mathrm{mg} / \mathrm{ml}$ mitomycin C (Sigma-Aldrich) for $2 \mathrm{~h}$ and seeded into the top wells at a density of $2.5 \times 10^{5}$ cells/well. For the rescue experiment, pDNA4-eNOS or its control plasmid was transfected into the cells. Invaded cells on the lower surface of the membrane were stained and counted.

Statistical Analysis. All data are presented as the mean \pm standard deviation. One-way analysis of variance was used to make comparisons between samples. $\mathrm{P}<0.05$ was considered to indicate a statistically significant difference. Statistical analysis was performed using SPSS version 19.0 (IBM SPSS, Inc, Armonk, NY, USA). All experiments were performed at least three times.

\section{Results}

Sample data. The expression levels of miR-146a/b, miR-21, miR-34a and miR-210 were measured and compared between two groups, the atherosclerotic plaque ('cases'; n=10) and normal control ('controls'; $n=10$ ) groups. No significant differences were identified between the cases and the controls with respect to age, gender, body mass index (BMI), blood pressure or glucose levels, as presented in Table I. Fig. 1A shows that the relative expression levels of miR-146a and miR-21 in atherosclerotic plaque samples was significantly upregulated to $320(\mathrm{P}<0.001)$ and $400 \%(\mathrm{P}<0.001)$, respectively, compared with the levels observed in normal controls, whereas the expression levels of miR-34a, miR-146b and miR-210 were similar between the two groups.

Identification of Notch2, instead of Notch1, as direct target genes of miR-146a in VSMCs. miR databases, including TargetScan (www.targetscan.org) and MIRDB (www.mirdb.org), were used in an attempt to identify the targets of miR-146a and miR-21. Notch1/Notch2 and Jag1 were identified as potential target genes of miR-146a and miR-21, respectively. The miRs and their seed sequences in the 3'-UTR of the candidate genes are presented in Fig. 2A and C. It was determined that overexpression of
Table II. Sequences of the quantitative reverse transcription polymerase chain reaction primer sets used for determination of microRNA expression levels.

\begin{tabular}{ll}
\hline Name & \multicolumn{1}{c}{ Sequences } \\
\hline miR-146a & 5'- ATTTTACAGGGCTGGGACAG-3' \\
miR-21 & 5'- TCTTCCAAGCTCTTCAGCAG-3' \\
& 5'- TGTCGGGTAGCTTATCAGAC-3' \\
miR-34a & 5'- TGTCAGACAGCCCATCGACT-3' \\
& 5'-TTGGCAGTGTCTTAGCTGGTT-3' \\
miR-210 & 5'-TTGCTGACCTCTGACCTTTTC-3' \\
& 5'- GACTGGCCTTTGGAAGCTCC-3' \\
miR-146b & 5'-ACAGCCTTTCTCAGGTGCAG-3' \\
& 5'- GGGCCTCAACTTACTCATCC-3' \\
U6 & 5'-GTTATTTAAAGGTTTGGCCAGT-3' \\
& 5'-CTCGCTTCGGCAGCACA-3' \\
& 5'-AACGCTTCACGAATTTGCGT-3' \\
\hline
\end{tabular}

the miR-146a mimic, but not the control mimics, substantially repressed the activity of luciferase fused with the 3'-UTR of Notch2, while having a minimal effect on the luciferase activity of Notch1, as shown in Fig. 1B. Simultaneously, a luciferase assay was performed, which revealed that the miR-21 mimic significantly inhibited the activity of luciferase fused with the 3'-UTR of Jag1 (P<0.001; Fig. 2D).

To further verify that Notch2 and Jag1 are functional target genes of miR-146a and miR-21 in HCASMCs, the cells were transfected with either an miR-146a mimic, an miR-21 mimic or control mimics, and the expression levels of Notch2 and Jag1 were determined by RT-PCR and western blot analysis. As shown in Fig. 3A, overexpression of miR-146a significantly inhibited the expression levels of Notch2 in HCASMCs, however, not those of Notch1 (results not shown). As Jag1 has been identified as a critical ligand of Notch, required for Notch to execute its function in regulating HCASMC proliferation, and Jag1 was additionally found to be a potential target gene of another highly expressed microRNA, miR-21 (R), the present study attempted to investigate whether Jag1 is a direct target of miR-21 and whether miR-21 affects the expression of Jag1 in HCASMCs. It was determined that transfection of HCASMCs 
A

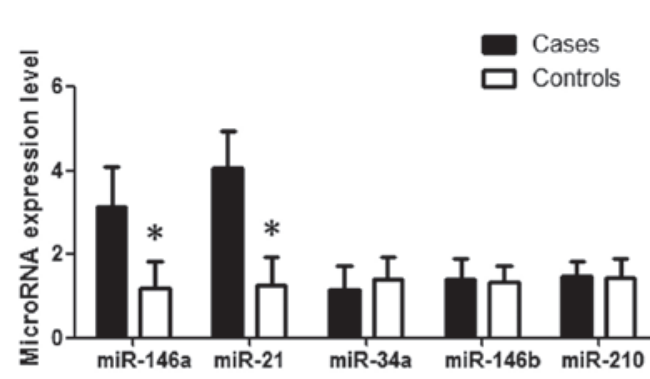

C

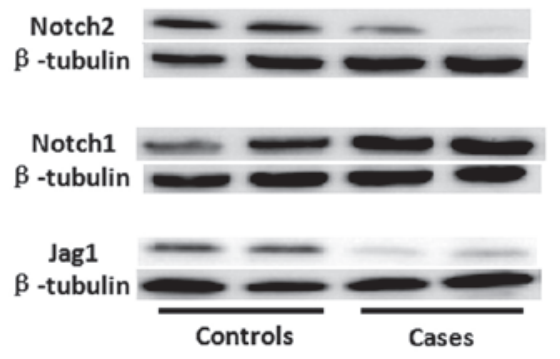

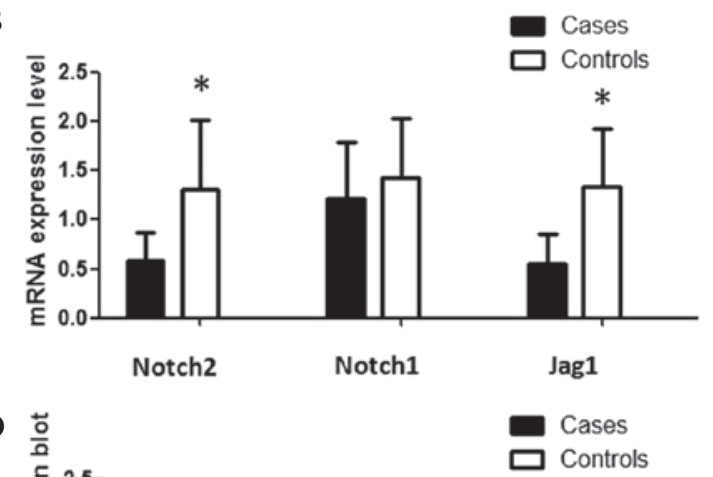

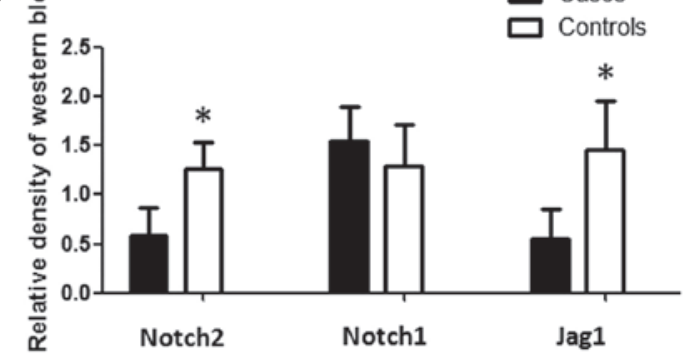

Figure 1. Comparison between atherosclerotic samples and the controls of (A) Expression patterns of miR-21, miR-34a, miR-146a, miR-210 and m146b. (B) mRNA expression levels of Notch1, Notch2 and Jag1. (C) Western blot analysis of protein expression levels of Notch1, Notch2 and Jag1. (D) Densitometric analysis and statistical comparison of each. A total of 10 cases and 10 controls tissue samples were included, and each experiment was performed in triplicate. " $\mathrm{P}<0.05$ vs. the corresponding cases measurement.

A

\begin{tabular}{|c|c|}
\hline \multicolumn{2}{|c|}{ A miR-146a targets Notch2 } \\
\hline $\operatorname{miR}-146 a-5 p \quad 3^{\prime}$ & $\begin{array}{c}\text { TTGGGTACCTTAA-GTCAAGAGU 5' } \\
\text { | }\|\|\|\mid\|\|\| \|\end{array}$ \\
\hline Notch2 & TTTTGGTTTGAATGACAGTTCTCA 3 ' \\
\hline $\operatorname{miR}-146 a-5 p 3^{\prime}$ & $\begin{array}{l}\text { TT----GGGTACCTTAAGTCAAGAGU } 5 \\
\|\quad\|\|\|\|\|\|\|\|\|\end{array}$ \\
\hline
\end{tabular}

miR-146a targets Not chl

miR-146a-5D $3^{\prime}$ TTGGGTACCTTAAGTCAAGAGU $5^{\prime}$ Notchl 5. CAAGAAAATAAACTAGTTCTCA 3

C
B

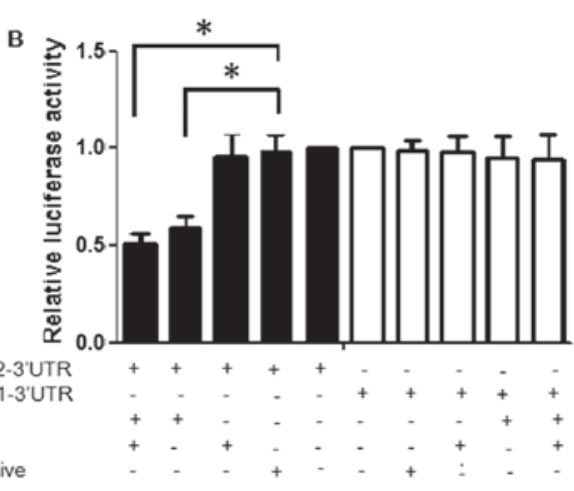

D

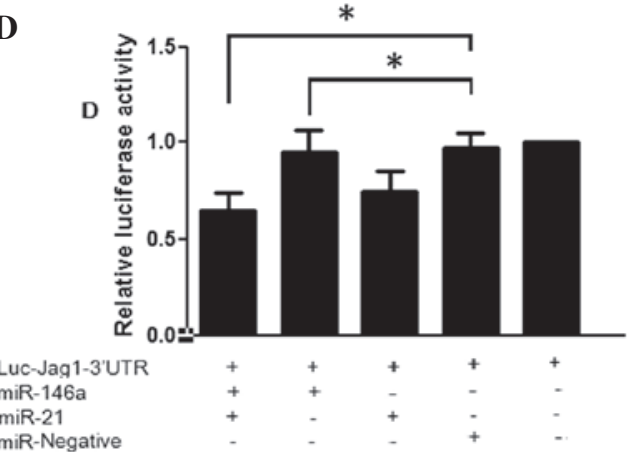

Figure 2. (A) Schematic representation of the miR-146a and miR-146a-binding sequence in the 3'-UTRs of Notch1 and Notch2 mRNA. (B) Analysis of luciferase activity in human coronary artery smooth muscle cells $48 \mathrm{~h}$ after co-transfection with the control Renilla luciferase expression construct pRL-TK and a firefly luciferase reporter plasmid containing the 3'-UTR of either Notch1 or Notch2. Firefly luciferase activity in each sample was normalized to Renilla activity as well as the luciferase activity of control cells. (C) Schematic representation of the miR-21 and miR-21-binding sequence in the 3'-UTR of the Jag1 mRNA; (D) Analysis of luciferase activity in human coronary artery smooth muscle cells $48 \mathrm{~h}$ after co-transfection with the control Renilla luciferase expression construct pRL-TK and a firefly luciferase reporter plasmid containing the 3'-UTR of Jag1. Firefly luciferase activity in each sample was normalized to the Renilla and firefly luciferase activity of the control cells. Each experiment was performed in triplicate. " $\mathrm{P}<0.05$. Luc, luciferase; miR, microRNA; UTR, untranslated region.

with an miR-21 mimic significantly suppressed the activity of luciferase fused with the 3'-UTR of Jag1 (P<0.001; Fig. 2D). Similar to the effect of miR-146a on Notch1 expression, overexpression of miR-21 markedly inhibited the expression of Jag1 in HCASMCs (Fig. 3B). In addition, Targetscan revealed Jag1 as a target of miR-34a, which was reported to be highly 
A

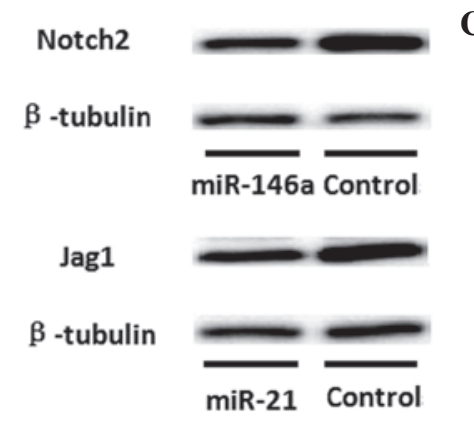

$\mathbf{C}$

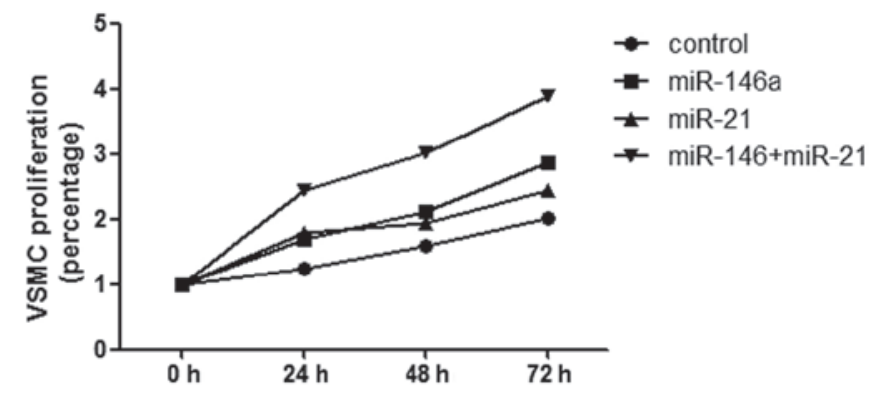

Figure 3. In vitro study of the proliferation of VSMCs transfected with miR-146a and/or miR-21 mimics. (A) Suppression of the protein expression levels of Notch2 and Jag1 by miR-146a and miR-21 mimics in human coronary artery smooth muscle cells. (B) Survival rate of HCASMCs transfected with miR-146a and/or miR-21 mimics, as determined by MTT assay 24, 48 and $72 \mathrm{~h}$ following transfection. (C) Promotion of proliferation of HCASMCs. HCASMCs, human coronary artery smooth muscle cells.

A

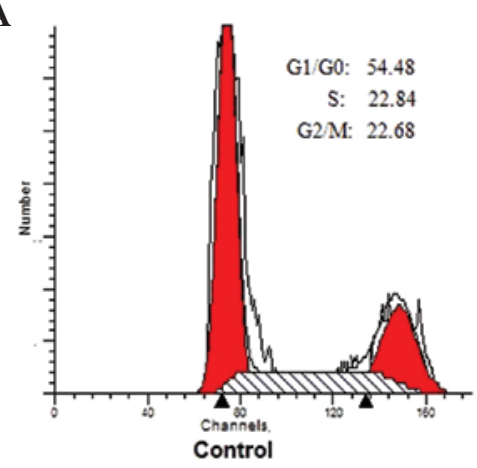

C

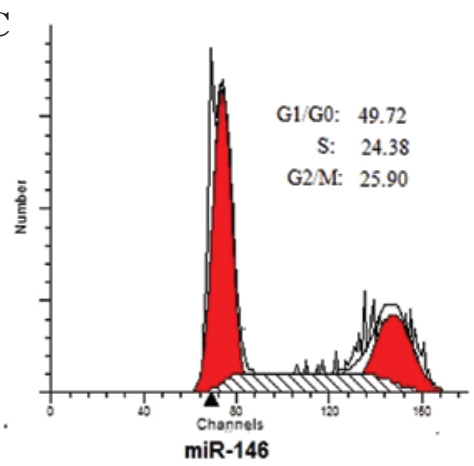

$\mathbf{E}$

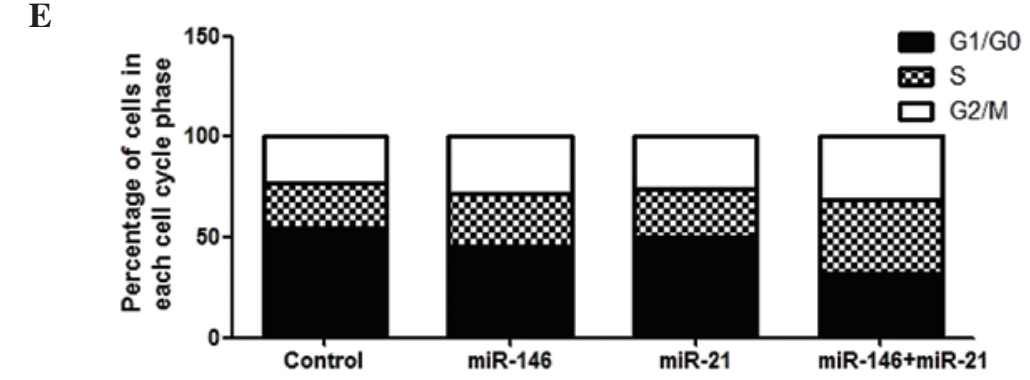

B

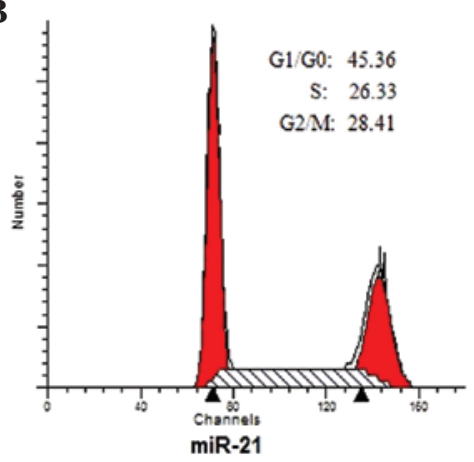

D

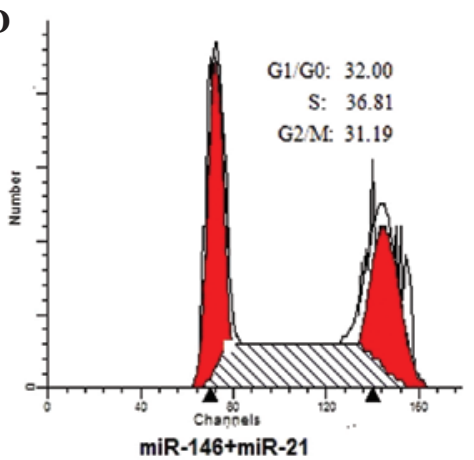

miR-146+miR-21

Figure 4. Effect of miR-146a and miR-21 mimics on cell cycle of HCASMCs. Flow cytometric analysis of HCASMCs transfected with (A) the controls, (B) miR-146a mimics, (C) miR-21 mimics and (D) miR-146a and miR-21 mimics. (E) Comparison of the percentage of the cells in each cell cycle phase in HCASMCs transfected with miR-146a, miR-21, miR-146a+miR-21 or the controls. HCASMCs, human coronary artery smooth muscle cells.

expressed in atherosclerosis with an 8-mer conserved site (18). However, the results of the present study found the expression levels of miR-34a were similar between the case and control samples (Fig. 1A); hence, its effect on the expression of Jag1 was not explored. Collectively, these results suggested that
miR-146a and miR-21 directly targeted Notch2 and Jag1, respectively, in order to cooperatively inhibit the Notch2/Jag1 signaling pathway in HCASMCs.

Furthermore, the expression patterns of the target genes of miR-146a and miR-21 were examined in the atherosclerotic 
samples and the controls, and it was revealed that the expression levels of Notch2 and Jag1 were substantially downregulated in atherosclerotic tissue samples compared with those of the controls (Fig. 1B and C).

Effects of miR-146a and miR-21 overexpression on VSMC proliferation and migration. HCASMCs were transfected with miR-146a mimics and an MTT assay was employed to measure cell proliferation. The results showed that exogenous expression of miR-146a significantly suppressed the expression levels of Notch2 (Fig. 3A), and substantially promoted proliferation of HCASMCs (Fig. 3C). Following incubation for $48 \mathrm{~h}$ and $72 \mathrm{~h}$, the levels of proliferation of the transfected cells were markedly higher than those of the controls, with the proliferation rates increased by $34 \%$ following incubation for $48 \mathrm{~h}(\mathrm{P}<0.01)$ and $42 \%$ following $72 \mathrm{~h}(\mathrm{P}<0.01)$, which suggests that the overexpression of miR-146a promotes the proliferation of HCASMCs in vitro. Similarly, transfection of the miR-21 mimic markedly suppressed the expression of Jag1 (Fig. 3B) and significantly enhanced the growth of HCASMCs, with the proliferation rates being increased by $22 \%$ following incubation for $48 \mathrm{~h}(\mathrm{P}<0.01)$ and $22 \%$ following incubation for $72 \mathrm{~h}$ $(\mathrm{P}<0.01)$, respectively (Fig. 3C). Additionally, HCASMCs were co-transfected with the mimics of miR-146a and miR-21, and the MTT assay was repeated to examine cell proliferation. It was determined that coexpression of miR-146a and miR-21 synergistically promoted cell proliferation, with the proliferation rate being increased by $90 \%$ following incubation for $48 \mathrm{~h}(\mathrm{P}<0.01)$ and 93\% following incubation for $72 \mathrm{~h}(\mathrm{P}<0.01)$, respectively. To evaluate the role of miR-146a and miR-21 in the regulation of VSMC migration, a migration assay was performed. The overexpression of miR-146a or miR-21 did not alter the migratory capability of HCASMCs (data not shown).

Effects of miR-146a and miR-21 overexpression on the cell cycle of the HCASMCs. Cell cycle analysis was performed to determine whether there was any cell cycle alteration in HCASMCs transfected with the mimics or the control. The results showed that compared with the control group, the $\mathrm{G}_{1}$ phase population of HCASMCs transfected with the miR-146a and miR-21 mimics was reduced from 54.48 to $45.36 \%$ (miR-146a alone, $\mathrm{P}<0.01$ ), from 54.48 to $49.72 \%$ (miR-21 alone, $\mathrm{P}<0.01$ ) and from 54.48 to $32.00 \%$ (miR-146a plus miR-21, $\mathrm{P}<0.01$ ), whereas the cells at the $\mathrm{S}$ phase increased from 22.84 to $26.23 \%$ (miR-146a alone, $\mathrm{P}<0.01$ ), from 22.84 to $24.38 \%$ (miR-21 alone, $\mathrm{P}<0.01$ ) and from 22.84 to $36.81 \%$ (miR-146a plus miR-21, $\mathrm{P}<0.01$ ). The population of cells in $\mathrm{G}_{2}$ phase increased from $22.68 \%$ to $28.41 \%$ (miR-146a alone, $\mathrm{P}<0.01$ ), from $22.68 \%$ to $25.90 \%$ (miR-21 alone, $\mathrm{P}<0.01$ ) and from $22.68 \%$ to 31.19 (miR-146a plus miR-21, $\mathrm{P}<0.01$ ), compared with that of the control group (Fig. 4A-E). These results indicated that overexpression of miR-146a and miR-21 synergistically induced $G_{1}$ phase cell cycle progression in HCASMCs and significantly increased the percentage of cells in the $\mathrm{S}$ and $\mathrm{G}_{2} / \mathrm{M}$ phases.

\section{Discussion}

miRs are considered to be involved in the regulation of biological and pathological cardiovascular activities (17).
Numerous miRs have been shown to have important roles in the regulation of VSMC growth and apoptosis. The levels of miR-146a were found to be significantly upregulated in rat balloon-injured arteries $(19,20)$, oxidized low-density lipoprotein-stimulated human monocytes/macrophages (21) and human atherosclerotic plaques (15). Lin et al (22) found that miR-21 protected VSMCs against apoptosis and participated in the $\mathrm{H}_{2} \mathrm{O}_{2}$-mediated vascular response by targeting activating protein-1 and programmed cell death protein 4. Mei et al (16) performed a thorough comparative screen using microarray analysis to identify the differentially expressed microRNAs in samples from atherosclerotic plaque and control VSMCs, and found miR-146a, miR-21, miR-34a and miR-128 as potential candidate miRs responsible for the differing vascular phenotype. The aim of the present study was to confirm the aberrant expression of the abovementioned four miRs in atherosclerotic plaque and control samples. The results demonstrated that the levels of miR-146a and miR-21 were consistently increased in atherosclerotic plaque compared with those in the controls, whereas the levels of miR-34a and miR-128 were comparable between the two groups.

According to the literature, miR-146a downregulates Notch1 in glioma cells and contributes to the pathogenesis of glioma (23), and Notch2 and Jag1 have been identified as potential target genes of miR-146a and miR-21. In the present study, the results of the luciferase assay confirmed that miR-146a and miR-21 suppress the expression of Notch2 and Jag1, respectively, whereas miR-146a did not alter the expression of Notch1 in VSMCs, as it did in glioma cells. Consistently, RT-PCR and western blot revealed that the overexpression of miR-146a and miR-21 downregulated the expression of Notch2 and Jag1, while having no effect on the expression of Notch1. Furthermore, the present study investigated the expression pattern of miR-146a, miR-21, Notch1, Notch2 and Jag1 in atherosclerotic and control cells. Compared with those in the normal controls, the expression levels of miR-146a and miR21 were significantly higher and the expression levels of Notch2 and Jag1 were lower in the atherosclerosis group, while the expression levels of Notch1 were similar.

VSMCs have been reported to produce extracellular matrices within the vessel wall in response to atherogenic stimuli (24), and to have an important role in the formation of the fibrous cap and plaque vulnerability in atherosclerosis (25). The accelerated proliferation of VSMCs causes accumulation of the cells and remodeling of the vessel wall. Thus, the deregulation of VSMC proliferation is key to the development of vascular diseases, including atherosclerosis and restenosis following balloon catheterization (26), and inhibition of VSMC proliferation may provide a novel therapeutic strategy for the treatment of such cardiovascular diseases (27). The Notch signaling pathway is highly conserved throughout evolution, and it is important in intercellular communication (28). To date, four Notch receptors and five ligands have been identified in mammals, which have been reported to have crucial roles in the regulation of a number of cellular activities, including differentiation, apoptosis and proliferation in a range of cell types during adult life (29). It has been shown that Notch1 promotes SMC proliferation and neointimal formation via the ligands $\mathrm{CHF} 1 / \mathrm{Hey} 2$, as evidenced by the transiently expressed Notch1 identified in neointimal SMCs following carotid artery 
ligation, whereas Notch2 expression was constitutive in both medial and neointimal SMCs (6). Lewis et al (8) showed that selectively enhancing Notch2 activity under the conditions of VSMC hyperproliferation may promote cell cycle exit. Notch2 is required for Jag-1-induced VSMC differentiation via the targeting of Skp2 and p27kip1 in order to reduce cell proliferation. A receptor-specific function for Notch2 was determined in the regulation of Jag-1-induced Skp2/p27kip1 expression and growth arrest in VSMCs by identifying colocalization of Notch2 and p27 kip1 to the nonproliferating region during vascular remodeling following carotid ligation, and a model was presented where Notch2 activation negatively regulates VSMC proliferation to lessen its severity (7).

In the present study it was hypothesized that Notch 2 and its ligand, Jag1, are transiently upregulated in, and provide crucial negative feedback on VSMC proliferation in response to vascular injury. As a negative regulator of VSMC proliferation, Notch2 forms a feedback loop with Notch1, an established positive regulator of VSMC proliferation, maintains a balance and controls the regulation of the acute reaction of VSMCs. Thus, in advanced atherosclerotic plaque, the highly expressed miR-146a specifically downregulates Notch2 and leaves Notch1 intact, which disturbs this balance and contributes to the formation of atherosclerotic plaque. In addition, due to the presence of highly expressed miR-21 in atherosclerotic plaque, a known negative regulator of Jag-1, the Notch2 pathway was further attenuated. These results confirmed that selectively enhancing miR-146a function promoted VSMC proliferation by targeting Notch2, and that the promoting effect could be reinforced by downregulating Jag1, a Notch2 ligand, necessary for its function in inducing cell cycle arrest, by overexpression of $\mathrm{miR}-21$, the other upregulated $\mathrm{miR}$ in atherosclerotic plaque.

In conclusion, the results of the present study showed that selective inhibition of the miRs that are significantly upregulated in atherosclerotic plaque may provide a novel strategy for preventing this disease. Thus, Notch 2 and its ligand are potential targets for the control of VSMC hyperplasia (7). The most widely used Notch antagonist is $\gamma$-secretase inhibitor, which is currently tested in clinical trials on patients with cancer (30).

\section{References}

1. Alexander MR and Owens GK: Epigenetic control of smooth muscle cell differentiation and phenotypic switching in vascular development and disease. Annu Rev Physiol 74: 13-40, 2012.

2. Carvalho FL, Simons BW, Eberhart CG and Berman DM: Notch signaling in prostate cancer: a moving target. Prostate 74 : 933-945, 2014

3. Fouillade C, Monet-Leprêtre M, Baron-Menguy C and Joutel A: Notch signalling in smooth muscle cells during development and disease. Cardiovasc Res 95: 138-146, 2012.

4. Doran AC, Meller N and McNamara CA: Role of smooth muscle cells in the initiation and early progression of atherosclerosis. Arterioscler Thromb Vasc Biol 28: 812-819, 2008.

5. Ottaviano FG, Handy DE and Loscalzo J: Redox regulation in the extracellular environment. Circ J 72: 1-16, 2008.

6. Li Y, Takeshita K, Liu PY, Satoh M, Oyama N, Mukai Y, Chin MT, Krebs L, Kotlikoff MI, Radtke F, Gridley T and Liao JK: Smooth muscle Notch1 mediates neointimal formation after vascular injury. Circulation 119: 2686-2692, 2009.

7. Boucher JM, Harrington A, Rostama B, Lindner V and Liaw L: A receptor-specific function for Notch2 in mediating vascular smooth muscle cell growth arrest through cyclin-dependent kinase inhibitor 1B. Circ Res 113: 975-985, 2013.
8. Lewis BP, Burge CB and Bartel DP: Conserved seed pairing, often flanked by adenosines, indicates that thousands of human genes are microRNA targets. Cell 120: 15-20, 2005.

9. Yu J, Wang F, Yang GH, Wang FL, Ma YN, et al: Human microRNA clusters: genomic organization and expression profile in leukemia cell lines. Biochem Biophys Res Commun 349: 59-68, 2006.

10. Brennecke J, Hipfner DR, Stark A, Russell RB and Cohen SM: bantam encodes a developmentally regulated microRNA that controls cell proliferation and regulates the proapoptotic gene hid in Drosophila. Cell 113: 25-36, 2003.

11. Calame K: MicroRNA-155 function in B Cells. Immunity 27: 825-827, 2007.

12. Jopling CL, Yi M, Lancaster AM, Lemon SM and Sarnow P: Modulation of hepatitis $\mathrm{C}$ virus RNA abundance by a liver-specific MicroRNA. Science 309: 1577-1581, 2005.

13. Cordes KR, Sheehy NT, White MP, Berry EC, Morton SU, Muth AN, Lee TH, Miano JM, Ivey KN and Srivastava D: miR-145 and miR-143 regulate smooth muscle cell fate and plasticity. Nature 460: 705-710, 2009.

14. Davis BN, Hilyard AC, Nguyen PH, Lagna G and Hata A: Induction of microRNA-221 by platelet-derived growth factor signaling is critical for modulation of vascular smooth muscle phenotype. J Biol Chem 284: 3728-3738, 2009.

15. Raitoharju E, Lyytikäinen LP, Levula M, Oksala N Mennander A, Tarkka M, Klopp N, Illig T, Kähönen M, Karhunen PJ, Laaksonen $\mathrm{R}$ and Lehtimäki T: miR-21, miR-210, miR-34a, and miR-146a/b are up-regulated in human atherosclerotic plaques in the Tampere Vascular Study. Atherosclerosis 219: 211-217, 2011.

16. Mei J, Bachoo R and Zhang CL: MicroRNA-146a inhibits glioma development by targeting Notch1. Mol Cell Biol 31: 3584-3592, 2011.

17. Urbich C, Kuehbacher A and Dimmeler S: Role of microRNAs in vascular diseases, inflammation, and angiogenesis. Cardiovasc Res 79: 581-588, 2008.

18. Staszel T,Zapała B, Polus A, Sadakierska-Chudy A, Kieć-Wilk B, Stepień E, Wybrańska I, Chojnacka M, Dembińska-Kieć A: Role of microRNAs in endothelial cell pathophysiology. Pol Arch Med Wewn 121: 361-366, 2011.

19. Ji R, Cheng Y, Yue J, Yang J, Liu X, Chen H, Dean DB and Zhang C: MicroRNA expression signature and antisense-mediated depletion reveal an essential role of MicroRNA in vascular neointimal lesion formation. Circ Res 8;100: 1579-88, 2007.

20. Sun SG, Zheng B, Han M, Fang XM, Li HX, Miao SB, Su M, Han Y, Shi HJ and Wen JK: miR-146a and Krüppel-like factor 4 form a feedback loop to participate in vascular smooth muscle cell proliferation. EMBO Rep 12: 56-62, 2011.

21. Chen T, Huang ZQ, Wang LS, Wang Y, Wu FZ, Meng S and Wang CQ: MicroRNA-125a-5p partly regulates the inflammatory response, lipid uptake, and ORP9 expression in oxLDL-stimulated monocyte/macrophages. Cardiovasc Res 83: 131-139, 2009.

22. Lin Y, Liu X, Cheng Y, Yang J, Huo Y, Zhang C: Involvement of microRNAs in hydrogen peroxide-mediated gene regulation and cellular injury response in vascular smooth muscle cells. J Biol Chem 284: 7903-7913, 2009.

23. Mei J, Bachoo R, Zhang CL: MicroRNA-146a inhibits glioma development by targeting Notch1. Mol Cell Biol 31: 3584-3592, 2011.

24. Ottaviano FG, Handy DE and Loscalzo J: Redox regulation in the extracellular environment. Circ J 72: 1-16, 2009.

25. Doran AC, Meller N and McNamara CA: Role of smooth muscle cells in the initiation and early progression of atherosclerosis. Arterioscler Thromb Vasc Biol 28: 812-819, 2008.

26. Inoue $\mathrm{T}$ and Node $\mathrm{K}$ : Molecular basis of restenosis and novel issues of drug-eluting stents. Circ J 73: 615-621, 2009.

27. Bornfeldt KE, Raines EW, Graves LM, Skinner MP, Krebs EG and Ross R: Platelet-derived growth factor: Distinct signal transduction pathways associated with migration versus proliferation. Ann NY Acad Sci 766: 416-430, 1995.

28. Alexander MR and Owens GK: Epigenetic control of smooth muscle cell differentiation and phenotypic switching in vascular development and disease. Annu Rev Physiol 74: 13-40, 2012.

29. Fouillade C, Monet-Leprêtre M, Baron-Menguy C and Joute A: Notch signalling in smooth muscle cells during development and disease. Cardiovasc Res 95:138-146, 2012.

30. Golde TE, Koo EH, Felsenstein KM, Osborne BA and Miele L: $\gamma$-Secretase inhibitors and modulators. Biochim Biophys Acta 1828: 2898-2907, 2013. 